\title{
Threshold-dependent iodine imaging and spectral separation in a whole-body photon-counting CT system
}

\author{
S. Sawall ${ }^{1,2}$ (D) L. Klein ${ }^{1,3} \cdot$ E. Wehrse ${ }^{2,4} \cdot$ L. T. Rotkopf ${ }^{2,4} \cdot$ C. Amato ${ }^{1,2} \cdot$ J. Maier ${ }^{1} \cdot$ H.-P. Schlemmer ${ }^{2,4} \cdot$ C. H. Ziener $^{2,4}$. \\ S. Heinze ${ }^{5}$ M. Kachelrieß ${ }^{1,2}$
}

Received: 30 November 2020 / Revised: 20 January 2021 / Accepted: 12 February 2021 / Published online: 13 March 2021

(C) The Author(s) 2021

\begin{abstract}
Objective To evaluate the dual-energy (DE) performance and spectral separation with respect to iodine imaging in a photoncounting CT (PCCT) and compare it to dual-source CT (DSCT) DE imaging.

Methods A semi-anthropomorphic phantom extendable with fat rings equipped with iodine vials is measured in an experimental PCCT. The system comprises a PC detector with two energy bins $(20 \mathrm{keV}, T)$ and $(T, \mathrm{e} U)$ with threshold $T$ and tube voltage $U$. Measurements using the PCCT are performed at all available tube voltages ( 80 to $140 \mathrm{kV})$ and threshold settings (50-90 keV). Further measurements are performed using a conventional energy-integrating DSCT. Spectral separation is quantified as the relative contrast media ratio $\mathrm{R}$ between the energy bins and low/high images. Image noise and dose-normalized contrast-to-noise ratio (CNRD) are evaluated in resulting iodine images. All results are validated in a post-mortem angiography study.

Results R of the PC detector varies between 1.2 and 2.6 and increases with higher thresholds and higher tube voltage. Reference $\mathrm{R}$ of the EI DSCT is found as 2.20 on average overall phantoms. Maximum CNRD in iodine images is found for $T=60 / 65 / 70 /$ $70 \mathrm{keV}$ for $80 / 100 / 120 / 140 \mathrm{kV}$. The highest CNRD of the PCCT is obtained using $140 \mathrm{kV}$ and is decreasing with decreasing tube voltage. All results could be confirmed in the post-mortem angiography study.

Conclusion Intrinsically acquired DE data are able to provide iodine images similar to conventional DSCT. However, PCCT thresholds should be chosen with respect to tube voltage to maximize image quality in retrospectively derived image sets.

Key Points

- Photon-counting CT allows for the computation of iodine images with similar quality compared to conventional dual-source dual-energy $C T$.

- Thresholds should be chosen as a function of the tube voltage to maximize iodine contrast-to-noise ratio in derived image sets.

- Image quality of retrospectively computed image sets can be maximized using optimized threshold settings.
\end{abstract}

Keywords Tomography, X-ray computed $\cdot$ Contrast media $\cdot$ Angiography $\cdot$ Iodine $\cdot$ Phantoms, imaging

\section{S. Sawall \\ stefan.sawall@dkfz.de}

1 Division of X-Ray Imaging and CT, German Cancer Research Center (DKFZ), Im Neuenheimer Feld 280, 69120 Heidelberg, Germany

2 Medical Faculty, Ruprecht-Karls-University Heidelberg, Im Neuenheimer Feld 672, 69120 Heidelberg, Germany

3 Department of Physics and Astronomy, Ruprecht-Karls-University Heidelberg, Im Neuenheimer Feld 226, 69120 Heidelberg, Germany

4 Division of Radiology, German Cancer Research Center (DKFZ), Im Neuenheimer Feld 280, 69120 Heidelberg, Germany

5 Institute of Forensic and Traffic Medicine, University Hospital Heidelberg, Voßstraße 2, 69115 Heidelberg, Germany

$\begin{array}{ll}\text { Abbreviations } \\ \text { CdTe } & \text { Cadmium telluride } \\ \text { CNR } & \begin{array}{l}\text { Contrast-to-noise ratio } \\ \text { CNRD }\end{array} \\ & \begin{array}{l}\text { Dose-normalized contrast-to-noise } \\ \text { ratio }\end{array} \\ \text { CT } & \text { Computed tomography } \\ \text { CTDI } & \text { Computed tomography dose index } \\ \text { DECT } & \text { Dual-energy CT } \\ \text { EI } & \text { Energy integrating } \\ \text { FOM } & \text { Field of measurement } \\ \text { FOV } & \text { Field of view } \\ \text { GOS } & \text { Gadolinium oxysulfide } \\ \text { PC } & \text { Photon counting } \\ \text { R } & \text { Relative contrast media ratio (RelCM) }\end{array}$


RelNoise Relative noise quotient

VMI Virtual monochromatic imaging

VNC Virtual non-contrast

\section{Introduction}

Over the last decades, computed tomography (CT) has proven to be an invaluable tool in clinical diagnostics. State-of-the-art systems allow for the acquisition of whole-body patient data in seconds and the resulting reconstructions provide a spatial resolution in the order of less than a millimeter. Besides conventional single energy imaging based on the integrated information from a single $\mathrm{x}$-ray spectrum, dual-energy CT (DECT) techniques have been introduced to clinical practice extending diagnostic capabilities even further. Popular applications include the computation of virtual monochromatic (VMI) and virtual non-contrast (VNC) images or material characterization and decomposition [1-3]. Various techniques have been proposed and are currently used in clinical CT systems to realize DECT. Most commonly, DECT is performed by using two distinct source-detector-pairs, rapid $\mathrm{kV}$-switching, duallayer sandwich detectors, or split filters [4-7]. Recently, prototypes of novel photon-counting (PC) detectors became available extending the range of dual- and multi-energy acquisition strategies. In the case of energy-integrating (EI) detectors, an incoming $\mathrm{x}$-ray photon is absorbed in the scintillator, typically $\mathrm{Gd}_{2} \mathrm{O}_{2} \mathrm{~S}$, resulting in the emission of optical photons that are detected in photo diodes eventually forming the desired signal. In the case of photon-counting detectors, the scintillator is replaced by a semiconductor, usually cadmium telluride (CdTe). The absorption of an incoming $\mathrm{x}$-ray photon results in the formation of a charge cloud that is transported to electrodes that are constituting the different detector pixels using a bias voltage in the order of $1 \mathrm{kV}$ [8-10]. The formed signals allow not only for the counting of single photons but also for the quantification of their energy. Typically, x-ray photons are sorted into two to four bins according to their energy, facilitating dual- or multi-energy acquisitions. In the case of two energy bins, a single threshold is used and photons with energy below this threshold are sorted to the lower bin and photons with energy above this threshold are sorted to the upper bin. This threshold can usually be placed almost arbitrarily with respect to the detected x-ray spectrum and influences the image quality of derived image sets, e.g., iodine maps or VMIs. Since the detectors always acquire at least two energy bins, spectral data are available even if no dedicated dual-energy acquisition was performed. A retrospective dual-energy evaluation of the acquired data might be desired, e.g., in the case of incidental findings or to retrospectively improve contrast agent enhancement. Thus, thresholds should always be set to maximize image quality even if a DECT evaluation is not within the initial scope of the examination. Hence, the influence of threshold position on the image quality of derived image sets, e.g., the iodine material maps, is of high interest and should be prospectively considered when setting up scan protocols [11]. This optimization problem shall be evaluated in this work. Hence, our focus is on contrast-enhanced imaging. The optimal threshold positions for other applications, e.g., virtual non-calcium images or imaging of potential other contrast agents [12,13], might differ given the spectral properties of the materials of interest. In particular, an experimental CT system equipped with a prototype photon-counting detector is used in all studies presented here. This detector has already proven to allow for dual- and multi-energy decomposition of conventional and potential novel contrast agents, to provide iodine quantification with high accuracy, and to allow for the computation of VMIs, among others, and several contrast-enhanced patient studies have been published [14-20]. We here evaluate the quality of derived iodine maps as a function of threshold, patient size, and tube voltage in a phantom study. Since the ionizing nature of $x$-rays prohibits multiple examinations of patients, the results will be verified in a post-mortem CT angiography study.

\section{Materials and methods}

\section{CT system}

Our experiments have been performed using the SOMATOM CounT experimental CT system (Siemens Healthineers, Fig. 1 left). This system is based on the SOMATOM Definition Flash dual-source CT whereas one of the conventional EI detectors was replaced by a PC detector allowing for a comparison of both technologies in a single system. The PC detector provides a native pixel size of $225 \mu \mathrm{m}$ but is operated in $4 \times 4$ binning mode referred to as "macro." This results in a pixel size of $0.50 \mathrm{~mm}$ in the iso-center which is close to the size of the energy-integrating detector of $0.6 \mathrm{~mm}$. Hence, the macro mode is the standard mode of operation provided by the experimental system. Measurements using the PC detector provide a field of measurement (FOM) of $275 \mathrm{~mm}$ while the EI detector in this system allows for acquisitions with a FOM of $500 \mathrm{~mm}$. Hence, patients and phantoms might exceed the FOM of the PC detector and a data completion scan using the EI detector is required to prevent degradation of image quality by truncation artifacts. This data completion scan would contribute to the overall dose. However, recent experiments illustrated that the required dose is similar to a single chest $\mathrm{x}$-ray [21]. Furthermore, the clinical version of a photon-counting CT might provide a sufficiently sized detector array and hence the data completion scan will be disregarded in the following. Unlike the EI detector, an operation of the PC detector in macro mode results in the acquisition of multi-energy data. 
Fig. 1 Left: Experimental photon-counting $\mathrm{CT}$ and the used semi-anthropomorphic phantom. Right: Semi-anthropomorphic liver phantom used in the experiments. The numbers indicate the iodine concentration in the vials measured in $\mathrm{mg} / \mathrm{mL}$. Zero denotes pure water

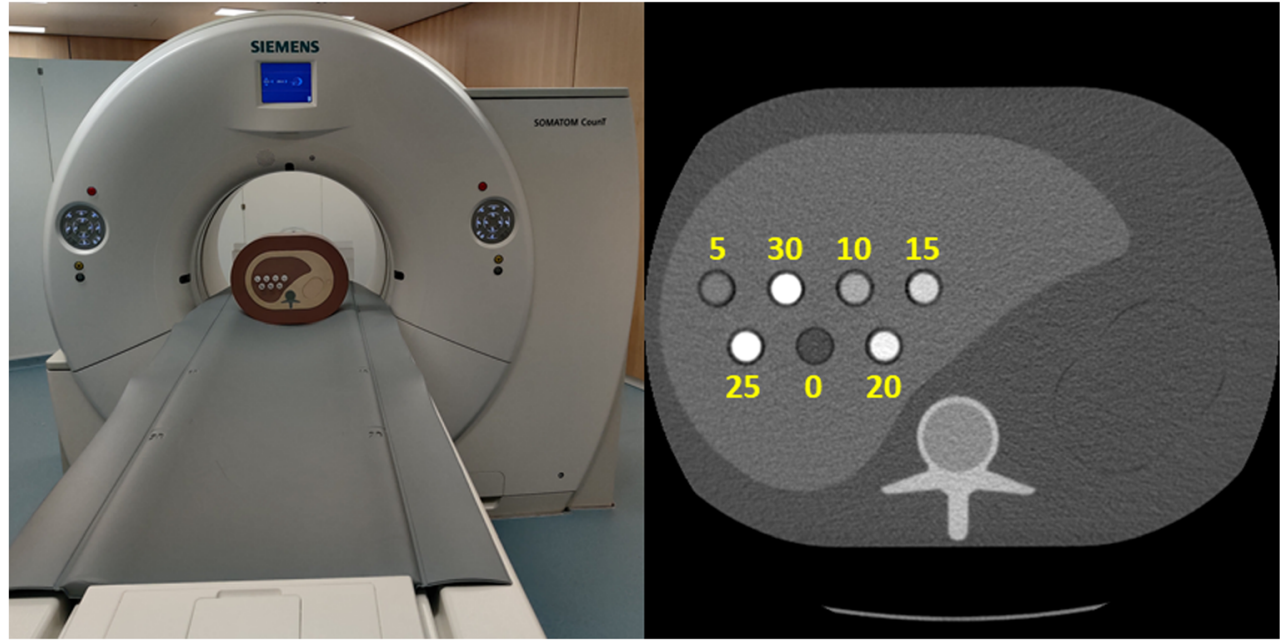

In particular, the detector simultaneously acquires two energy bins with the lower bin covering an energy range of $\left(T_{1}, T_{2}\right)$ and the higher bin covering $\left(T_{2}, e U\right)$, with $U$ being the tube voltage and $e$ being the elementary charge, while also providing images covering the whole spectrum, i.e., $\left(T_{1}, e U\right)$. Reconstructed images of the energy bins will be referred to as $f_{\text {Low }}$ for the lower bin and $f_{\text {High }}$ for the upper bin, whereas $f$ denotes the image reconstructed using the entire spectrum. The thresholds $T_{1 / 2}$ can be chosen almost arbitrarily between 20 and $50 \mathrm{keV}$ for $T_{1}$, and 50 and $90 \mathrm{keV}$ for $T_{2}$. The threshold $T_{1}$ will be fixed at $20 \mathrm{keV}$, and hence, $T_{2}$ will only be referred to as $T$ in the following.

\section{Material decomposition}

Let us consider $\mathrm{CT}$ images normalized to air at 0 and water at 1 [22]. Given the reconstructed image of the lower energy bin $f_{\text {Low }}$ and the image of the high energy bin $f_{\text {High }}$, one might compute a water image $f_{\mathrm{W}}$, i.e., a VNC image, as

$f_{W}=(1-\beta) f_{\text {Low }}+\beta f_{\text {High }}$

Here, $\beta=R /(R-1)$ is a weighting coefficient wherein $\beta=\beta(U, T)$ is a function of the tube voltage $U$ and threshold $T$. The scalar $R$ is the relative contrast media ratio (RelCM) and a measure for the spectral separation between the images $f_{\text {Low }}$ and $f_{\text {High }}$ [23]. It is sometimes also referred to as CT number ratio or dual-energy ratio [24]. I.e., given two wateriodine mixtures of unknown mixing ratio, one can show that $R$ is independent of the mixing ratio and is only a function of the $\mathrm{CT}$ values of iodine in these images as [22]

$R=C T_{\text {Low }} / C T_{\text {High }}$

In practice, $C T_{\text {Low }}$ and $C T_{\text {High }}$ are the mean $\mathrm{CT}$ values measured in regions of interest containing diluted contrast media, e.g., the aorta or portal vein, and can be used to calibrate DECT. The variance of $f_{\mathrm{W}}$ is
$\operatorname{Var} f_{W}=(1-\beta)^{2} \operatorname{Var} f_{\text {Low }}+\beta^{2} \operatorname{Var} f_{\text {High }}$.

The noise in the VNC image is thus $\sigma_{f_{W}}=\sqrt{\operatorname{Var} f_{W}}$ which is a function of the variance and thus of the noise in $f_{\text {Low }}$ and $f_{\text {High }}$. Similar to a VNC image, an iodine image $f_{\mathrm{I}}$ can be computed as

$f_{I}=\gamma\left(f_{\text {Low }}-f_{\text {High }}\right)$.

The variance of this iodine map is

$\operatorname{Var} f_{I}=\gamma^{2} \operatorname{Var} f_{\text {Low }}+\gamma^{2} \operatorname{Var} f_{\text {High }}$

The scalar $\gamma$ is chosen such that $f_{\mathrm{I}}+f_{\mathrm{W}}=\mathrm{f}_{\alpha}$ with $f_{\alpha}$ being a mixed image with minimum noise [25], i.e.,

$f_{\alpha}=(1-\alpha) f_{\text {Low }}+\alpha f_{\text {High }}$.

The scalar $\alpha=\operatorname{Var} f_{\text {Low }} /\left(\operatorname{Var} f_{\text {Low }}+\operatorname{Var} f_{\text {High }}\right)$ is chosen according to an inverse variance weighting that minimizes the noise in the resulting image $f_{\alpha}$. Given its dependence on $f_{\alpha}$ and $f_{\mathrm{W}}$, the iodine image is also a function of tube voltage, threshold, and noise in $f_{\text {Low }}$ and $f_{\text {High. }}$. In the case of conventional dual-source dual-energy imaging, the noise in $f_{\text {Low }}$ and $f_{\text {High }}$ can be adapted separately by adjusting the tube current for the two different $\mathrm{x}$-ray sources. $R$ remains unaffected as long as the tube voltages are unaltered. Hence, the optimal image in terms of noise and CNRD is found for balanced noise levels between both tubes. In the case of photon-counting CT with a single detector, noise in $f_{\text {Low }}$ and $f_{\text {High }}$ is a function of the threshold and cannot be adjusted separately. Furthermore, changing the threshold changes not only the image noise but also the detected spectrum in each bin and hence $R$. Thus, the lowest noise in the iodine image might not be found for balanced noise levels between both bins.

Note that in practice, $f_{\mathrm{W}}$ and $f_{\mathrm{I}}$ usually undergo a variety of vendor-specific post-processing steps to compensate for the 
increase in noise due to the material decomposition. However, only the raw material maps prior to any post-processing shall be evaluated and presented in the following to exclude any potential benefits or drawbacks of the used de-noising methods.

\section{Phantom experiments}

All phantom experiments presented in the following have been performed using a semi-anthropomorphic liver phantom (QRM) that can be extended with fat rings to mimic different patient sizes. In particular, a small-sized (S, $20 \mathrm{~cm} \times 30 \mathrm{~cm})$, a medium-sized (M, $25 \mathrm{~cm} \times 35 \mathrm{~cm})$, and a large-sized patient $(\mathrm{L}, 30 \mathrm{~cm} \times 40 \mathrm{~cm})$ were modelled. All phantoms were equipped with vials containing iodine solutions with concentrations between 5 and $30 \mathrm{mg} / \mathrm{mL}$ (see Fig. 1). Image acquisition was performed using all available tube voltages, namely $80 \mathrm{kV}, 100 \mathrm{kV}, 120 \mathrm{kV}$, and $140 \mathrm{kV}$ with a constant dose level $D$ of $11 \mathrm{mGy}\left(\mathrm{CTDI}_{32 \mathrm{~cm}}\right)$ allowing for a comparison of results at unit dose. The detector's energy threshold was varied in steps of $5 \mathrm{keV}$ between $50 \mathrm{keV}$ and the maximum available threshold for a respective tube voltage. In particular, the maximum available thresholds were $70 \mathrm{keV}$ for a tube voltage of $80 \mathrm{kV}, 80 \mathrm{keV}$ for a tube voltage of $100 \mathrm{kV}$, and $90 \mathrm{keV}$ for tube voltages of $120 \mathrm{kV}$ and $140 \mathrm{kV}$, respectively. Image reconstruction was performed using weighted filtered backprojection covering the entire FOV of $275 \mathrm{~mm}$ using a D40f-kernel onto a matrix of $512 \times 512$ pixels resulting in a pixel size of $0.54 \mathrm{~mm}$ and with a slice thickness of $1.50 \mathrm{~mm}$ and a slice increment of $0.75 \mathrm{~mm}$. $R$ was evaluated using ROIs placed in the iodine insert containing $15 \mathrm{mg} / \mathrm{mL}$. Iodine images were computed according to Eq. (4) and the contrast-tonoise ratio at unit dose $(\mathrm{CNRD})$ was measured as

$\mathrm{CNRD}=\frac{\left|c_{1}-c_{2}\right|}{\sqrt{\sigma_{1}^{2}+\sigma_{2}^{2}}} \cdot \frac{1}{\sqrt{D}}$.

Therein, $c_{\mathrm{i}}$ are iodine concentrations measured in the 15 $\mathrm{mg} / \mathrm{mL}$ iodine insert and the background and $\sigma_{i}$ are the corresponding standard deviations measured in these ROIs. Since all acquisitions have been performed at the same dose level, a normalization of the CNR with respect to dose is not required. However, we prefer to report CNRD to illustrate that it is a function of dose in general. To investigate the influence of image noise in general and the balancing of noise between individual bins, we define the noise quotient (RelNoise) as follows:

RelNoise $=\frac{\sigma_{\text {Low }}}{\sigma_{\text {High }}}$.

Reference measurements were conducted using an energyintegrating dual-source dual-energy CT system (Definition Flash) using tube voltage pairs of $80 \mathrm{kV} / \mathrm{Sn} 140 \mathrm{kV}$ and 100
$\mathrm{kV} / \mathrm{Sn} 140 \mathrm{kV}$ with $\mathrm{Sn}$ denoting an additional 0.4-mm tin prefilter to improve the spectral separation. The tube currents were adjusted to match the radiation dose level used at the photon-counting CT. The FOM of the DSCT is $500 \mathrm{~mm}$ and $330 \mathrm{~mm}$ for the first and second imaging chains, respectively. To match the pixel size of reconstructions obtained using the PC detector, reconstructions of the DSCT cover a FOV of 275 $\mathrm{mm}$. A summary of all relevant acquisition and reconstruction parameters can be found in Table 1 .

\section{Post-mortem CT angiography study}

Given the ionizing radiation used in CT, experiments similar to the phantom measurements cannot be performed in patients since multiple acquisitions would be required. To overcome this drawback and to illustrate that the results obtained using phantoms can be translated to clinical practice, a post-mortem CT angiography study was performed after approval by the local ethics committee (S-021/2020). This study follows published and accepted protocols [26, 27]. In brief, a cadaver was placed in supine position on the patient table and the right femoral vessels were cannulated. About 3.5 L of an iodinebased contrast medium was injected with a flow rate of about $800 \mathrm{~mL} / \mathrm{min}$. The oily nature of the contrast agent (AngiofilMacro, Fumedica AG and paraffin oil) and its optimized viscosity prevent a high loss of perfusate into the surrounding tissue and are reported to prevent rapid tissue edematization [28]. Images were acquired using a tube voltage of $140 \mathrm{kV}$ and a dose of $11 \mathrm{mGy}$ using the PC detector. The threshold was varied between 50 and $90 \mathrm{keV}$ in steps of $5 \mathrm{keV}$. Iodine images were computed according to Eq. 4. Comparative acquisitions at the DSCT similar to the phantom experiments could not be conducted in the presented post-mortem study due to the contrast agent kinetics. I.e., a transport of the cadaver to the DSCT after PC acquisitions, increasing extravasation of contrast media over time, and an accumulation of contrast media at the bottom of vessels due to the lack of circulation alter the distribution of contrast media significantly and do not allow for a fair side-by-side comparison of both technologies as shown in the phantom experiments.

\section{Results}

\section{Spectral separation}

Figure 2 shows $R$ for all available tube voltages obtained in the insert containing $15 \mathrm{mg} \mathrm{I} / \mathrm{mL}$. Colored, solid lines in this figure represent different phantom sizes, i.e., the Sphantom (green), the M-phantom (blue), and the Lphantom (red). For each tube voltage, the threshold was varied in steps of $5 \mathrm{keV}$ between $50 \mathrm{keV}$ and the maximum possible value for the respective tube voltage. Black 
Table 1 Acquisition and reconstruction parameters for all experiments conducted with phantoms and the post-mortem angiography study (FOM, field of measurement; $F O V$, field of view; $A E C$, automatic exposure control)

\begin{tabular}{|c|c|c|}
\hline & DSCT & PCCT \\
\hline Tube voltage $e_{\text {phantoms }}$ & $80 \mathrm{kV} / 140 \mathrm{kV}+\mathrm{Sn}, 100 \mathrm{kV} / 140 \mathrm{kV}+\mathrm{Sn}$ & $80 \mathrm{kV}, 100 \mathrm{kV}, 120 \mathrm{kV}, 140 \mathrm{kV}$ \\
\hline Tube voltage $e_{\text {post-mortem }}$ & - & $140 \mathrm{kV}$ \\
\hline $\mathrm{CTDI}_{32 \mathrm{~cm}}$ & $11 \mathrm{mGy}$ & $11 \mathrm{mGy}$ \\
\hline $\mathrm{AEC}$ & off & off \\
\hline FOM & $500 \mathrm{~mm} / 330 \mathrm{~mm}$ & $275 \mathrm{~mm}$ \\
\hline FOV & $275 \mathrm{~mm}$ & $275 \mathrm{~mm}$ \\
\hline Image matrix & $512 \times 512 \mathrm{px}$ & $512 \times 512 \mathrm{px}$ \\
\hline Pixel size & $0.54 \mathrm{~mm}$ & $0.54 \mathrm{~mm}$ \\
\hline Slice thickness & $1.50 \mathrm{~mm}$ & $1.50 \mathrm{~mm}$ \\
\hline Slice increment & $0.75 \mathrm{~mm}$ & $0.75 \mathrm{~mm}$ \\
\hline Kernel & D40f & D40f \\
\hline
\end{tabular}

dashed-dotted lines show $R$ measured in an energy-integrating, dual-energy CT and represent the average overall phantom sizes. In the case of the photon-counting detector, $R$ steadily increases with an increasing threshold. E.g., in the case of $140 \mathrm{kV}, R$ is about 1.5 for a threshold of $50 \mathrm{keV}$ and rises to about 2.5 at a threshold of $90 \mathrm{keV}$. This holds for all investigated tube voltages. At any given threshold, $R$ increases with increasing tube voltage. E.g., for a threshold of $60 \mathrm{keV}$, a tube voltage of $80 \mathrm{kV}$ results in a $R$ of about 1.4 , and in 1.6 using $100 \mathrm{kV}, 1.65$ for
$120 \mathrm{kV}$ and finally in 1.76 using $140 \mathrm{kV}$. The reference DECT system achieves relative contrast media ratios of about 2.8 for $80 \mathrm{kV} / \mathrm{Sn} 140 \mathrm{kV}$ and about 2.2 for 100 $\mathrm{kV} / \mathrm{Sn} 140 \mathrm{kV}$ which is consistent with values already published in the literature [23]. Hence, the reference system generally shows a higher relative contrast media ratio compared to the investigated photon-counting detector. Only higher thresholds and tube voltages of $120 \mathrm{kV}$ and $140 \mathrm{kV}$, respectively, exhibit similar values to the 100 $\mathrm{kV} / \mathrm{Sn} 140 \mathrm{kV}$ reference protocol.
Fig. $2 R$ as function of threshold for all available tube voltages. Colors encode phantom sizes, i.e., the S-phantom (green), the Mphantom (blue), and the Lphantom (red). Black dasheddotted lines show $R$ measured in a reference dual-source dual-energy CT system and represent the average overall phantom sizes
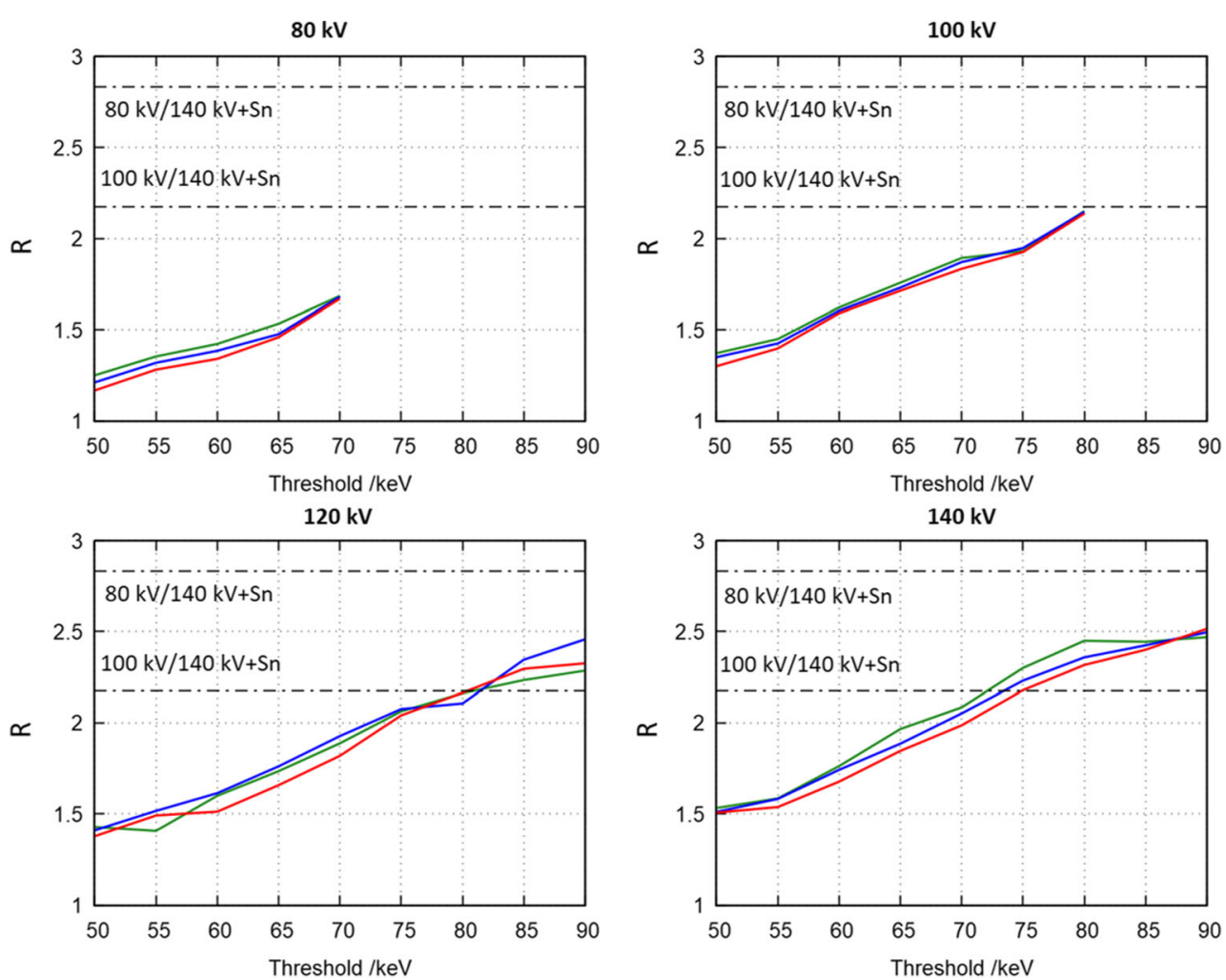


\section{Material decomposition}

Figure 3 shows the CNRD (see Eq. 7, colored solid lines) for all available tube voltages using thresholds varied in $5-\mathrm{keV}$ steps similar to Fig. 2. The line colors encode the phantom sizes. Black solid lines show the CNRD obtained using the reference DECT system using the S-phantom. Furthermore, the figure shows RelNoise (see Eq. 8, dashed lines). In general, CNRD decreases with increasing phantom size and increasing intersection lengths, due to the increase in image noise. All CNRD plots obtained using the investigated photon-counting detector show distinct maxima. In the case of the S-phantom, the optimal CNRD using a tube voltage of $80 \mathrm{kV}$ is found as $0.71 \mathrm{mGy}^{-1 / 2}$ at a threshold of $60 \mathrm{keV}$. For $100 \mathrm{kV}$, the optimal CNRD of $0.84 \mathrm{mGy}^{-1 / 2}$ is achieved at a threshold of $65 \mathrm{keV}$ and tube voltages of $120 \mathrm{kV}$ and $140 \mathrm{kV}$ show maxima at $70 \mathrm{keV}$ with CNRDs of $0.98 \mathrm{mGy}^{-1 / 2}$ and $1.04 \mathrm{mGy}^{-1 / 2}$, respectively. While CNRD decreases with increasing phantom size, the position of the optimal threshold remains unchanged. This holds for all investigated tube voltages and all phantom sizes. The noise quotient RelNoise illustrates that unlike conventional DECT, maximum CNRD is not found for balanced background noise levels between bins. In particular, maximum CNRD is found for a noise quotient of 0.63 in the case of $80 \mathrm{kV}, 0.72$ for $100 \mathrm{kV}, 0.73$ for $120 \mathrm{kV}$, and 0.74 for 140 $\mathrm{kV}$, respectively. The noise quotient does not show a dependence on phantom size. As indicated in Fig. 2, the used reference system achieves higher CNRDs of $1.29 \mathrm{mGy}^{-1 / 2}$ and 1.99
$\mathrm{mGy}^{-1 / 2}$ for the protocols $80 \mathrm{kV} / \mathrm{Sn} 140 \mathrm{kV}$ and $100 \mathrm{kV} / \mathrm{Sn} 140$ $\mathrm{kV}$. Previous studies illustrated that the root-mean-square errors between ground truth iodine concentrations and the ones measured in material maps are similar between the reference DECT system and the experimental system equipped with a photon-counting detector [11].

The results presented in Fig. 3 are further visualized in Fig. 4. The figure shows iodine material maps obtained of the Sphantom for all available tube voltages acquired at the optimal threshold and acquired at the same dose level. It is evident that acquisitions and material decomposition with $80 \mathrm{kV}$ result in the highest noise among all acquisitions. The lowest noise and highest CNRD for the photon-counting detector are found at $140 \mathrm{kV}$ and using a threshold of $70 \mathrm{keV}$.

\section{Post-mortem CT angiography study}

To illustrate that the phantom results can be translated to clinical practice, Fig. 5 shows reconstructions obtained in a postmortem CT angiography study. The figures show measurements conducted at $140 \mathrm{kV}$ using thresholds of $50 \mathrm{keV}, 70$ $\mathrm{keV}$, and $90 \mathrm{keV}$. The tube voltage of $140 \mathrm{kV}$ was chosen since it results in the highest CNRD according to previous sections. The top row of Fig. 5 shows the low energy bin, the second row the high energy bin, and the bottom row the iodine material map. All rows include a magnified region of interest including the right hepatic vein and the inferior caval vein. An increase of the threshold resulted in a noise reduction
Fig. 3 Dose-normalized contrastto-noise ratio (CNRD) of iodine maps (colored solid lines) and noise quotient (RelNoise $=\sigma_{\text {Low }} /$ $\sigma_{\text {High }}$, dashed lines) for all phantoms sizes, all tube voltages, and all thresholds. Colors encode phantom sizes, i.e., the Sphantom (green), the M-phantom (blue), and the L-phantom (red). Black dashed-dotted lines were acquired using the M-phantom in a reference dual-source dual-energy CT system
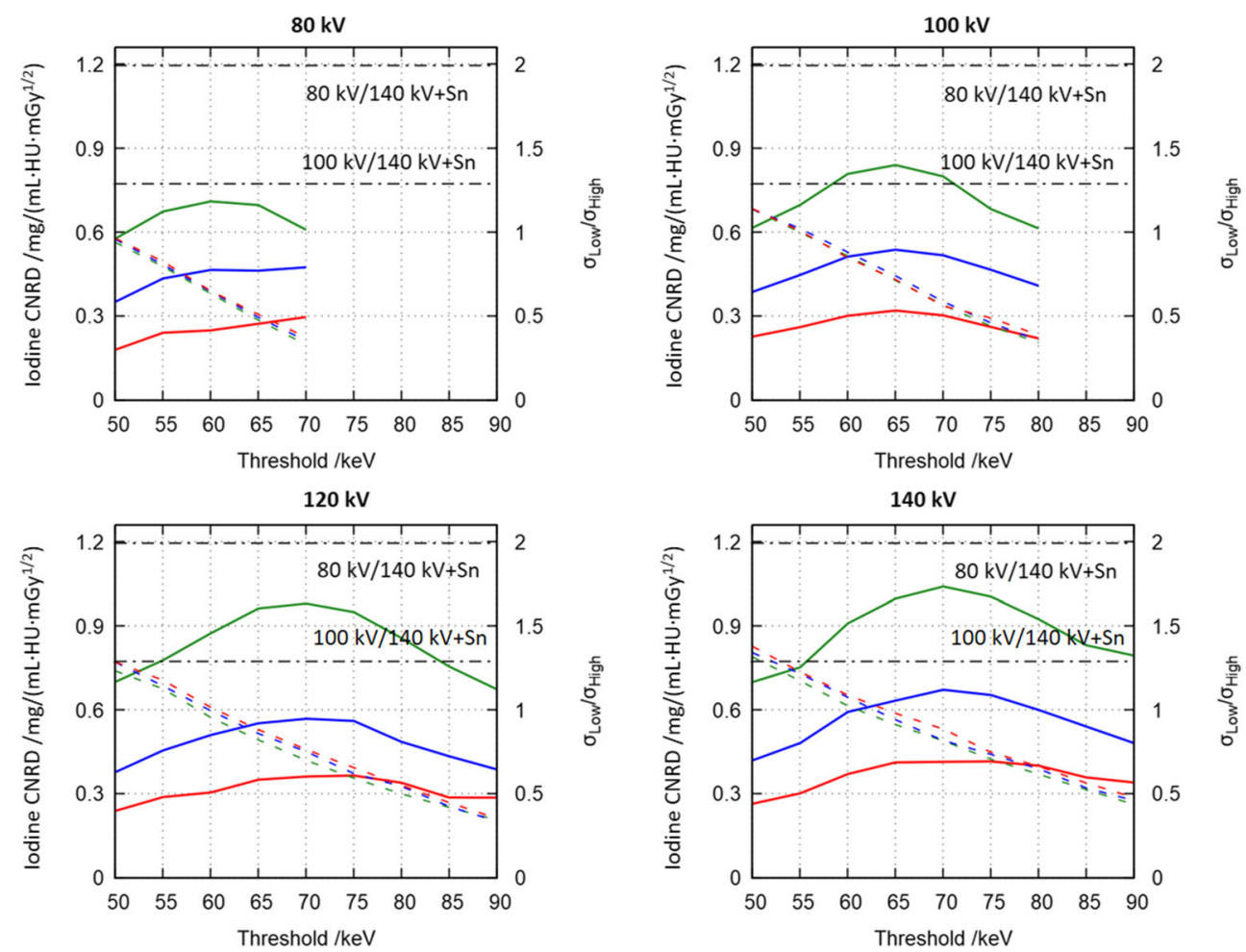
Fig. 4 Iodine maps for all available tube voltages of the Sphantom acquired at the same dose. Images were acquired at the optimal threshold according to Fig. 3. Magnifications of the yellow dashed region of interest are shown in the top right of each figure $(C=7 \mathrm{mg} / \mathrm{mL}, W=30 \mathrm{mg}$ ) $\mathrm{mL})$

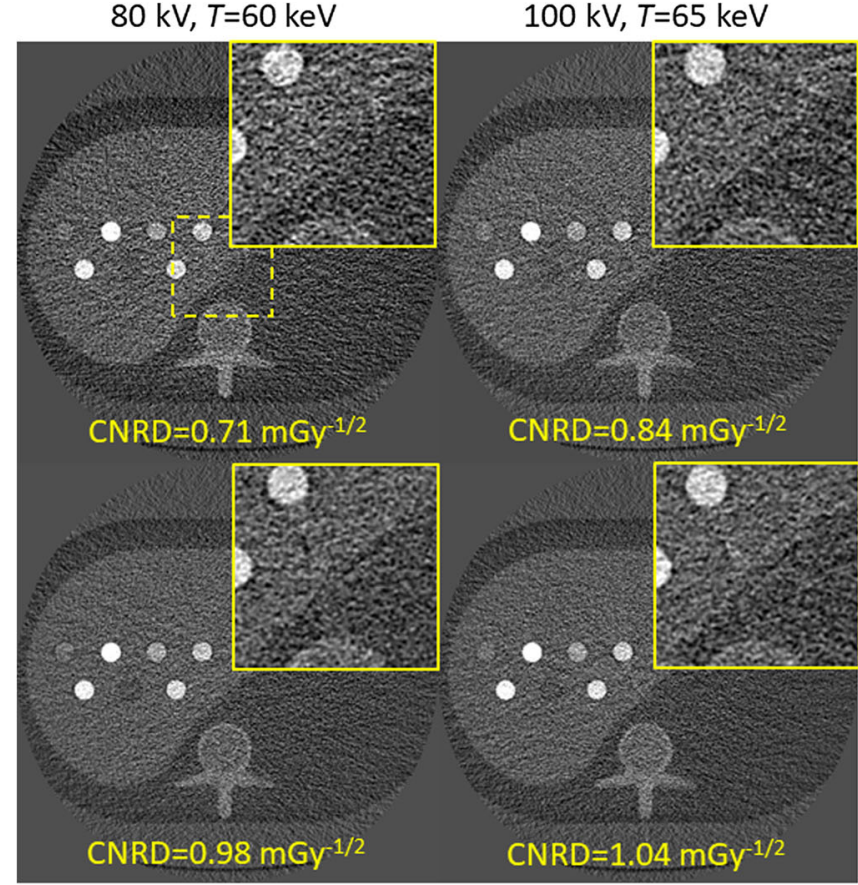

$140 \mathrm{kV}, T=70 \mathrm{keV}$

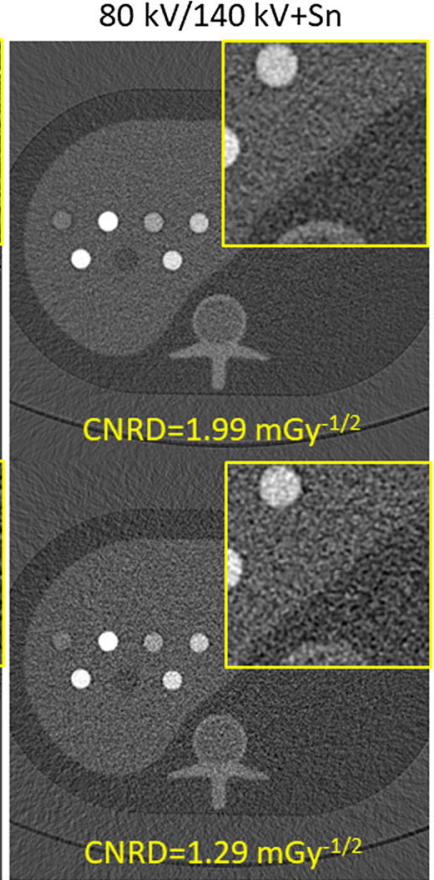

$100 \mathrm{kV} / 140 \mathrm{kV}+\mathrm{Sn}$ in the lower energy bin and in an increase in noise in the higher energy bin. I.e., using a threshold of $50 \mathrm{keV}$ resulted in a noise level of $34 \mathrm{HU}$ in the lower bin and in $23 \mathrm{HU}$ in the higher bin. Increasing the threshold to $70 \mathrm{keV}$ decreases noise in the lower bin to $25 \mathrm{HU}$ and increases noise in the higher bin to $31 \mathrm{HU}$, while the noise is $24 \mathrm{HU}$ and $45 \mathrm{HU}$ in these bins at $90 \mathrm{keV}$. Since increasing the threshold increases the effective energy of the detected $\mathrm{x}$-ray spectrum in both bins, contrast is reduced. This is particularly evident in the high energy bin and the iodine contrast in the larger vessels visualized therein. Similar to the results presented in previous sections, the best CNRD in the iodine material maps can be achieved using a threshold of $70 \mathrm{keV}$ as $2.17 \mathrm{mGy}^{-1 / 2}$ confirming the results presented in Figs. 3 and 4. Thresholds above or below this optimum show lower CNRDs of $1.30 \mathrm{mGy}^{-1 / 2}$ using a threshold of $50 \mathrm{keV}$ and $1.96 \mathrm{mGy}^{-1 / 2}$ using a threshold of $90 \mathrm{keV}$.

\section{Discussion}

Our study shows that the threshold of photon-counting detectors should be set according to the used tube voltage to ensure that image quality in material maps of iodine in terms of noise and CNRD is maximized. Since the investigated scan mode of the photon-counting detector always acquires two energy bins simultaneously, this also applies to standard examinations that are usually not intended as dual-energy examinations, but might be subject to a material decomposition retrospectively in case of accidental findings. Figure 2 illustrates that the relative contrast media ratio, a measure for the spectral separation between acquired bin data, is a function of the used threshold and increases with an increase thereof. Hence, if the noise in the low and high energy bin was constant, the best image quality would be achieved at the highest available threshold. However, since a change in threshold also affects the detected bin spectra and image noise, iodine CNRD shows distinct maxima as a function of tube voltage. This was illustrated using phantom measurements and in a post-mortem CT angiography study. Unlike in conventional dual-energy CT, the best image quality in the iodine maps was not achieved for a balanced image noise between bins, but for noise ratios between 0.6 and 0.8 (see Fig. 3). A comparison to iodine maps obtained using a conventional, energyintegrating dual-source dual-energy system revealed that the relative contrast media ratio of the investigated photon-counting detector is similar to the one observed in this system. However, given that the threshold cannot be changed without changing image noise and relative contrast media ratio, the image quality of the investigated photon-counting detector is slightly inferior to the reference system. This is consistent with the observations in the literature [29]. Other authors proposed the usage of dualsource photon-counting systems equipped with two photoncounting detectors to overcome this fact [30]. However, such systems do not yet exist and the cost-efficient future system might only be equipped with a single detector. The experiments presented herein assume a detector with two distinct energy bins. Other scan modes or detectors might provide even more simultaneously acquired energy bins. The used detector for example also provides a mode that acquires four bins simultaneously. However, $\mathrm{R}$ is not a suitable metric to describe the spectral separation between more than two energy bins. The design of 

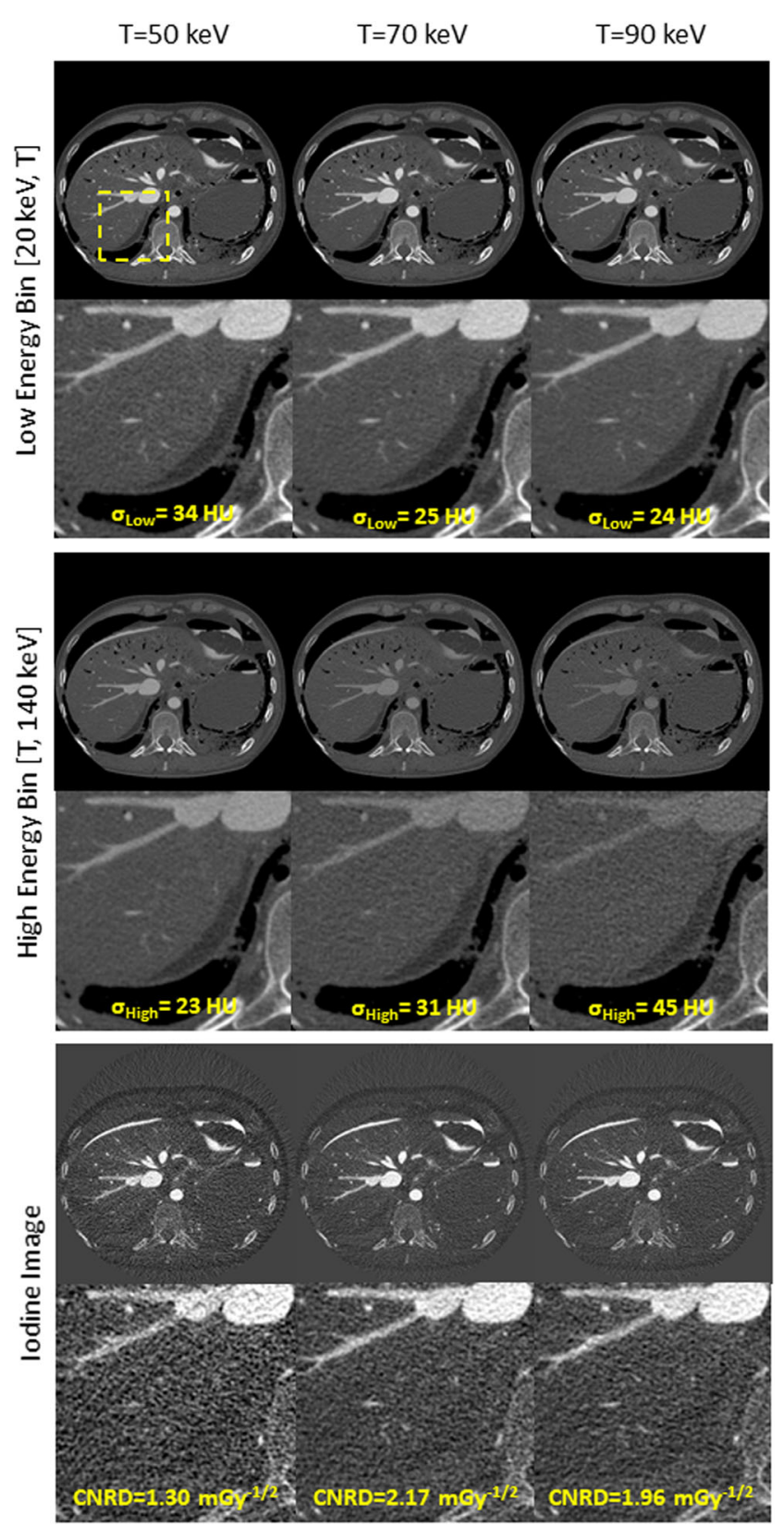

Fig. 5 Post-mortem CT angiography using a tube voltage of $140 \mathrm{kV}$ for thresholds of $50 \mathrm{keV}$ (left column), $70 \mathrm{keV}$ (middle column), and $90 \mathrm{keV}$ (right column). Low energy bins (top row), high energy bins (middle row), and iodine maps (bottom row) (bin images $C=300 \mathrm{HU}, W=$ $1400 \mathrm{HU}$; material maps $C=15 \mathrm{mg} / \mathrm{mL}, W=30 \mathrm{mg} / \mathrm{mL}$ )

suitable metrics for systems with more than two energy bins and the actual number of bins required to maximize e.g. the CNRD in iodine maps is a topic of ongoing research [31]. Photon-counting detectors not only allow for the simultaneous acquisition of multiple energy bins but also provide a variety of other benefits. Popular examples include but are not limited to a reduction in radiation and contrast media dose [32-34]. Hence, our experiments provide guidelines for threshold settings maximizing image quality in iodine maps, even if a dual-energy decomposition is only performed retrospectively.
Acknowledgements The authors would like to thank Sebastian Faby and Christoph Polster (Siemens Healthineers) for their valuable support in conducting this study.

Funding Open Access funding enabled and organized by Projekt DEAL.

\section{Declarations}

Guarantor The scientific guarantor of this publication is Prof. Marc Kachelrieß.

Conflict of interest The authors of this manuscript declare no relationships with any companies whose products or services may be related to the subject matter of the article.

Statistics and biometry No complex statistical methods were necessary for this paper.

Informed consent Written informed consent was waived by the Institutional Review Board.

Ethical approval Institutional Review Board approval was obtained.

\section{Methodology \\ - experimental}

Open Access This article is licensed under a Creative Commons Attribution 4.0 International License, which permits use, sharing, adaptation, distribution and reproduction in any medium or format, as long as you give appropriate credit to the original author(s) and the source, provide a link to the Creative Commons licence, and indicate if changes were made. The images or other third party material in this article are included in the article's Creative Commons licence, unless indicated otherwise in a credit line to the material. If material is not included in the article's Creative Commons licence and your intended use is not permitted by statutory regulation or exceeds the permitted use, you will need to obtain permission directly from the copyright holder. To view a copy of this licence, visit http://creativecommons.org/licenses/by/4.0/.

\section{References}

1. Albrecht MH, Vogl TJ, Martin SS et al (2019) Review of clinical applications for virtual monoenergetic dual-energy CT. Radiology 293:260-271. https://doi.org/10.1148/radiol.2019182297

2. Toepker M, Moritz T, Krauss B et al (2012) Virtual non-contrast in second-generation, dual-energy computed tomography: reliability of attenuation values. Eur J Radiol 81:e398-e405. https://doi.org/ 10.1016/j.ejrad.2011.12.011

3. Patino M, Prochowski A, Agrawal MD et al (2016) Material separation using dual-energy CT: current and emerging applications. Radiographics 36:1087-1105. https://doi.org/10.1148/rg. 2016150220

4. Faby S, Kuchenbecker S, Sawall S et al (2015) Performance of today's dual energy $\mathrm{CT}$ and future multi energy $\mathrm{CT}$ in virtual non-contrast imaging and in iodine quantification: a simulation study. Med Phys 42:4349-4366. https://doi.org/10.1118/1. 4922654

5. Euler A, Parakh A, Falkowski AL et al (2016) Initial results of a single-source dual-energy computed tomography technique using a 
split-filter: assessment of image quality, radiation dose, and accuracy of dual-energy applications in an in vitro and in vivo study. Invest Radiol 51:491-498. https://doi.org/10.1097/RLI. 0000000000000257

6. Lell MM, Wildberger JE, Alkadhi H, Damilakis J, Kachelriess M (2015) Evolution in computed tomography: the battle for speed and dose. Invest Radiol 50:629-644. https://doi.org/10.1097/RLI. 0000000000000172

7. Lell MM, Kachelrieß M (2020) Recent and upcoming technological developments in computed tomography: high speed, low dose, deep learning, multienergy. Invest Radiol 55:8-19. https://doi.org/ 10.1097/RLI.0000000000000601

8. Shikhaliev PM, Xu T, Molloi S (2005) Photon counting computed tomography: concept and initial results: photon counting computed tomography. Med Phys 32:427-436. https://doi.org/10.1118/1. 1854779

9. Shikhaliev PM (2008) Computed tomography with energyresolved detection: a feasibility study. Phys Med Biol 53:14751495. https://doi.org/10.1088/0031-9155/53/5/020

10. Taguchi K, Iwanczyk JS (2013) Vision 20/20: Single photon counting $\mathrm{x}$-ray detectors in medical imaging. Med Phys 40: 100901. https://doi.org/10.1118/1.4820371

11. Zhou W, Abdurakhimova D, Bruesewitz M, Halaweish A, McCollough CH, Leng S (2018) Impact of photon counting detector technology on $\mathrm{kV}$ selection and diagnostic workflow in CT. In: Chen G-H, Lo JY, Gilat Schmidt T (eds) Medical imaging 2018: physics of medical imaging. SPIE, Houston, $\mathrm{p} 47$

12. Amato C, Klein L, Wehrse E et al (2020) Potential of contrast agents based on high- $Z$ elements for contrast-enhanced photoncounting computed tomography. Med Phys:mp.14519. https://doi. org/10.1002/mp.14519

13. Müller FC, Børgesen H, Gosvig K et al (2019) Optimising dualenergy CT scan parameters for virtual non-calcium imaging of the bone marrow: a phantom study. Eur Radiol Exp 3:46. https://doi. org/10.1186/s41747-019-0125-2

14. Kappler S, Henning A, Krauss B et al (2013) Multi-energy performance of a research prototype CT scanner with small-pixel counting detector. In: Nishikawa RM, Whiting BR, Hoeschen C (eds) Medical Imaging 2013: Physics of Medical Imaging. https:// doi.org/10.1117/12.2006747

15. Pourmorteza A, Symons R, Sandfort V et al (2016) Abdominal imaging with contrast-enhanced photon-counting CT: first human experience. Radiology 279:239-245. https://doi.org/10.1148/ radiol.2016152601

16. Leng S, Zhou W, Yu Z et al (2017) Spectral performance of a whole-body research photon counting detector CT: quantitative accuracy in derived image sets. Phys Med Biol 62:7216-7232. https:// doi.org/10.1088/1361-6560/aa8103

17. Tao S, Rajendran K, McCollough CH, Leng S (2019) Feasibility of multi-contrast imaging on dual-source photon counting detector (PCD) CT: an initial phantom study. Med Phys 46:4105-4115. https://doi.org/10.1002/mp.13668

18. Fetterly KA, Michalak GJ, Zhou W et al (2018) Determination of optimal image type and lowest detectable concentration for iodine detection on a photon counting detector-based multi-energy CT system. In: Chen G-H, Lo JY, Gilat Schmidt T (eds) Medical Imaging 2018: Physics of Medical Imaging. SPIE, Houston, p 180

19. Ren L, Rajendran K, Fletcher JG, McCollough CH, Yu L (2020) Simultaneous dual-contrast imaging of small bowel with iodine and bismuth using photon-counting-detector computed tomography: a feasibility animal study. Invest Radiol. https://doi.org/10.1097/RLI. 0000000000000687

20. Symons R, Krauss B, Sahbaee P et al (2017) Photon-counting CT for simultaneous imaging of multiple contrast agents in the abdomen: an in vivo study. Med Phys 44:5120-5127. https://doi. org/10.1002/mp.12301

21. Yu Z, Leng S, Li Z et al (2016) How low can we go in radiation dose for the data-completion scan on a research whole-body photon-counting computed tomography system. J Comput Assist Tomogr 40:663-670. https://doi.org/10.1097/RCT. 0000000000000412

22. Dorn S, Chen S, Sawall S et al (2018) Towards context-sensitive CT imaging - organ-specific image formation for single (SECT) and dual energy computed tomography (DECT). Med Phys 45: 4541-4557. https://doi.org/10.1002/mp.13127

23. Krauss B, Grant KL, Schmidt BT, Flohr TG (2015) The importance of spectral separation: an assessment of dual-energy spectral separation for quantitative ability and dose efficiency. Invest Radiol 50: 114-118. https://doi.org/10.1097/RLI.0000000000000109

24. Primak AN, Giraldo JCR, Eusemann CD et al (2010) Dual-source dual-energy CT with additional tin filtration: dose and image quality evaluation in phantoms and in vivo. AJR Am J Roentgenol 195: 1164-1174. https://doi.org/10.2214/AJR.09.3956

25. Yu L, Primak AN, Liu X, McCollough CH (2009) Image quality optimization and evaluation of linearly mixed images in dualsource, dual-energy CT. Med Phys 36:1019-1024. https://doi.org/ $10.1118 / 1.3077921$

26. Saunders SL, Morgan B, Raj V, Rutty GN (2011) Post-mortem computed tomography angiography: past, present and future. Forensic Sci Med Pathol 7:271-277. https://doi.org/10.1007/ s12024-010-9208-3

27. Gascho D, Thali MJ, Niemann T (2018) Post-mortem computed tomography: technical principles and recommended parameter settings for high-resolution imaging. Med Sci Law 58:70-82. https:// doi.org/10.1177/0025802417747167

28. Grabherr S, Grimm J, Baumann P, Mangin P (2015) Application of contrast media in post-mortem imaging (CT and MRI). Radiol Med 120:824-834. https://doi.org/10.1007/s11547-015-0532-2

29. Kappler S, Niederlöhner D, Stierstorfer K, Flohr T (2010) Contrastenhancement, image noise, and dual-energy simulations for quantum-counting clinical CT. San Diego, California, United States, $\mathrm{p} 76223 \mathrm{H}$

30. Tao A, Huang R, Tao S, Michalak GJ, McCollough CH, Leng S (2019) Dual-source photon counting detector CT with a tin filter: a phantom study on iodine quantification performance. Phys Med Biol 64:115019. https://doi.org/10.1088/1361-6560/ab1c34

31. Taguchi K (2020) The number of energy windows for photon counting detectors: is more actually more? In: Proceedings of The 6th International Meeting on Image Formation in X-Ray Computed Tomography (CT-Meeting 2020). pp 422-425

32. Sawall S, Klein L, Amato C et al (2020) Iodine contrast-to-noise ratio improvement at unit dose and contrast media volume reduction in whole-body photon-counting CT. Eur J Radiol 126:108909. https://doi.org/10.1016/j.ejrad.2020.108909

33. Klein L, Dorn S, Amato C et al (2020) Effects of detector sampling on noise reduction in clinical photon-counting whole-body computed tomography. Invest Radiol 55:111-119. https://doi.org/10.1097/ RLI.0000000000000616

34. Hsieh SS, Leng S, Rajendran K, et al (2020) Photon counting CT: clinical applications and future developments. IEEE Trans Radiat Plasma Med Sci 1-1. https://doi.org/10.1109/TRPMS.2020. 3020212

Publisher's note Springer Nature remains neutral with regard to jurisdictional claims in published maps and institutional affiliations. 